\title{
Early satiety and postprandial fullness in gastroparesis correlate with gastroparesis severity, gastric emptying, and water load testing
}

\author{
H.P. Parkman ${ }^{1}$ | E. K. Hallinan ${ }^{2}$ | W. L. Hasler ${ }^{3}$ | G. Farrugia ${ }^{4}$ ～K. L. Koch ${ }^{5}$ | \\ L. Nguyen ${ }^{6}$ | W. J. Snape ${ }^{7}$ | T. L. Abell ${ }^{8}$ | R. W. McCallum ${ }^{9}$ | I. Sarosiek ${ }^{9}$ | \\ P. J. Pasricha
2 J. Clarke $^{2}$ (D) | L. Miriel ${ }^{2}$ | J. Tonascia ${ }^{2}$ | F. Hamilton ${ }^{10}$ | \\ The NIDDK Gastroparesis Clinical Research Consortium (GpCRC)
}

${ }^{1}$ Temple University, Philadelphia, PA, USA

${ }^{2}$ Johns Hopkins University, Baltimore, MD, USA

${ }^{3}$ University of Michigan, Ann Arbor, MI, USA

${ }^{4}$ Mayo Clinic, Rochester, MN, USA

${ }^{5}$ Wake Forest University, Winston-Salem, NC, USA

${ }^{6}$ Stanford University, Palo Alto, CA, USA

${ }^{7}$ California Pacific Medical Center, San

Francisco, CA, USA

${ }^{8}$ University of Louisville, Louisville, KY, USA

${ }^{9}$ Texas Tech University, El paso, TX, USA

${ }^{10}$ National Institute of Diabetes and Digestive and Kidney Diseases, Bethesda, MD, USA

\section{Correspondence}

Henry P. Parkman, MD, Gastroenterology Section, Parkinson Pavilion, Temple University School of Medicine, Philadelphia, PA, USA.

E-mail: henry.parkman@temple.edu

Funding information

National Institute of Diabetes and Digestive and Kidney Diseases (NIDDK), Grant/Award Number: U01DK073975, U01DK073983, U01DK073985, U01DK074007, U01DK073974 and U01DK074008

\begin{abstract}
Background: Early satiety (ES) and postprandial fullness (PPF) are often present in gastroparesis, but the importance of these symptoms in gastroparesis has not been well-described. The aims were: (i) Characterize ES and PPF in patients with gastroparesis. (ii) Assess relationships of ES and PPF with etiology of gastroparesis, quality of life, body weight, gastric emptying, and water load testing.

Methods: Gastroparetic patients filled out questionnaires assessing symptoms (PAGISYM) and quality of life (PAGI-QOL, SF-36v2). Patients underwent gastric emptying scintigraphy and water load testing.

Key Results: 198 patients with gastroparesis (134 IG, 64 DG) were evaluated. Early satiety was severe or very severe in $50 \%$ of patients. Postprandial fullness was severe or very severe in $60 \%$ of patients. Severity scores for ES and PPF were similar between idiopathic and diabetic gastroparesis. Increasing severity of ES and PPF were associated with other gastroparesis symptoms including nausea/vomiting, satiety/early fullness, bloating, and upper abdominal pain and GERD subscores. Increasing severity of ES and PPF were associated with increasing gastroparesis severity, decreased BMI, decreased quality of life from PAGI-QOL and SF-36 physical health. Increasing severity of ES and PPF were associated with increasing gastric retention of a solid meal and decreased volume during water load test.

Conclusions \& Inferences: Early satiety and PPF are commonly severe symptoms in both diabetic and idiopathic gastroparesis. Early satiety and PPF severity are associated with other gastroparesis symptom severities, body weight, quality of life, gastric emptying, and water load testing. Thus, ES and PPF are important symptoms characterizing gastroparesis. ClinicalTrials.gov number: NCT NCT01696747.
\end{abstract}

KEYWORDS

diabetic gastroparesis, early satiety, gastric emptying, gastroparesis 


\section{1 | INTRODUCTION}

Symptoms of gastroparesis can be varied, but classically include nausea and vomiting. ${ }^{1}$ Early satiety (ES) and postprandial fullness (PPF) can also be present. Early satiety and PPF have been reported to be present in $54-60 \%$ of patients with gastroparesis. ${ }^{2,3}$ The Gastroparesis Cardinal Symptom Index-Daily Diary (GCSI-DD) developed through patient interviews and input from the Federal Drug Administration (FDA) contains five symptoms: nausea, vomiting, ES, PPF, and abdominal pain. ${ }^{4,5}$ Both ES and PPF were included in this patient reported outcome. They appear to capture different pathophysiologic aspects of gastroparesis: ES due to impaired proximal gastric (fundic) function and PPF due to impaired distal gastric (antral) function.

Normally with solid food ingestion, the upper stomach relaxes, allowing the proximal stomach to accommodate the ingested meal. This is followed by a progressively tonic contraction to deliver food into the distal stomach. ${ }^{6-8}$ In the antrum, regular peristaltic contractions grind down solid food so that it can be passed out the pylorus. With impaired gastric accommodation, there is increased pressure in the upper stomach after meal ingestion compromising the ability of the upper stomach to act as a reservoir for ingested food., 10 Impaired accommodation has shown to be associated with ES and weight loss in patients with functional dyspepsia. ${ }^{10}$ Impaired proximal gastric function has also been described in patients with idiopathic gastroparesis $^{11}: 25$ of 58 patents (43\%) patients with gastroparesis had impaired accommodation using a gastric barostat, and this was associated with higher prevalence of ES and weight loss.

Postprandial fullness has been associated with delayed gastric emptying which is primarily due to impaired antral function. While smaller studies have found inconsistent associates of symptoms to delayed gastric emptying, larger scale studies often report association of delayed gastric emptying with increased severity of PPF, nausea, and vomiting. ${ }^{12}$ For examples, in two studies (one of 343 patients and another of 392 patients with functional dyspepsia seen in referral centers), the symptoms of PPF and vomiting were associated with delayed gastric emptying of solids. ${ }^{13,14}$

Thus, EF and PPF appear to be important symptoms in patients with gastroparesis. The attributes and importance of these symptoms and effects on patients have not been specifically characterized. The aims of this study were to characterize ES and PPF in patients with gastroparesis. We wanted to assess relationships of ES and PPF with etiology of gastroparesis, quality of life, body weight, gastric emptying, and water load testing.

\section{2 | METHODS}

\section{1 | Overview}

The NIDDK Gastroparesis Clinical Research Consortium (GpCRC) is a cooperative network of eight academic motility centers and one Data Coordinating Center (DCC) funded through the NIDDK of the

\section{Key Points}

- Early satiety (ES) and postprandial fullness (PPF) are often present in gastroparesis, but the importance of these symptoms in gastroparesis has not been well-described.

- ES and PPF are commonly severe symptoms in both diabetic and idiopathic gastroparesis. ES and PPF severity are associated with other gastroparesis symptom severities, body weight, quality of life, gastric emptying, and water load testing.

- ES and PPF are important symptoms characterizing gastroparesis.

National Institutes of Health (NIH). ${ }^{15,16}$ The Gastroparesis Registry 2 (ClinicalTrials.gov Identifier: NCT NCT01696747) was implemented as an observational study of patients with gastroparesis enrolled prospectively. This study uses data from the second gastroparesis registry (GpR2) of the NIDDK GpCRC. GpR2 was designed, in part, to enhance the understanding of symptoms and physiologic dysfunction in patients with gastroparesis. Physiologic testing included measuring gastric emptying of liquids in the presence of solids, and performing a water load test.

This report focuses on patients with either idiopathic or diabetic gastroparesis. The diabetic patients could have either Type 1 diabetes mellitus (T1DM) or type 2 diabetes mellitus (T2DM) as defined by the patient and their physician. The diagnosis of patients with the idiopathic etiology was based on no previous gastric surgery, no diabetes history (before or after the onset of gastroparesis at enrollment), a normal hemoglobin $\mathrm{A} 1_{C}$, and no other known etiologies.

All studies were approved by the Institutional Review Board at each Clinical Center and at the DCC. The authors had access to the study data and have reviewed and approved the final manuscript.

\section{2 | Enrollment}

Gastroparetic patients were enrolled at eight centers into the $\mathrm{NIH}$ Gastroparesis Registry from September 2012 to March 2016. Enrolled patients met specific entry criteria being 18 years or older with symptoms of at least 12 weeks duration, delayed gastric emptying scintigraphy (GES) using the 4 hour Eggbeaters protocol (gastric retention $>60 \%$ at 2 hours and/or $>10 \%$ at 4 hours) within 6 months of enrollment, and no structural abnormality as seen by upper endoscopy within 1 year of enrollment.

\section{3 | Study protocol}

During face-to-face interviews with each subject, the study physicians or coordinators completed case report forms including data relating to gastroparesis disease onset, symptoms, disease profile, associated medical conditions, medication, and supplemental therapies. Study physicians performed a comprehensive physical examination. 
Laboratory measures were obtained, including hemoglobin $A 1_{C}$ values, antinuclear antibody (ANA), and erythrocyte sedimentation rate (ESR).

The clinical severity of gastroparesis was graded on a scale originally proposed by Abell et al. and reported in the American Neurogastroenterology and Motility Society (ANMS) review on treatment of gastroparesis. ${ }^{17}$ The severity was graded as grade 1: mild gastroparesis (symptoms relatively easily controlled and able to maintain weight and nutrition on a regular diet); grade 2: compensated gastroparesis (moderate symptoms with only partial control with use of daily medications, able to maintain nutrition with dietary adjustments); grade 3: gastroparesis with gastric failure (refractory symptoms that are not controlled as shown by the patient having ER visits, frequent doctor visits or hospitalizations and/or inability to maintain nutrition via an oral route).

Each patient filled out the 20 item Patient Assessment of Upper Gastrointestinal Symptoms (PAGI-SYM) questionnaire which assesses symptoms of gastroparesis, dyspepsia, and gastroesophageal reflux disease ${ }^{18}$; it includes the nine symptoms of the GCSI which asks about nausea, retching, vomiting, stomach fullness, inability to finish meal, excessive fullness, loss of appetite, bloating, and abdominal distension. ${ }^{19}$ Early satiety was assessed using the phrase "Not able to finish a normal-sized meal (for a healthy person)." Postprandial fullness was assessed with the phrase "Feeling excessively full after meals." The PAGI-SYM also inquires about symptoms of gastroesophageal reflux including daytime heartburn, heartburn lying down, daytime chest discomfort, nighttime chest discomfort, daytime reflux, nighttime reflux, and bitter taste. In the PAGI-SYM, patients are asked to assess the severity of their symptoms during the previous 2 weeks using a 0-5 scale where no symptoms $=0$, very mild $=1$, mild $=2$, moderate $=$ 3 , severe $=4$, and very severe $=5$. The GCSI equals the mean of the nausea/vomiting subscore, PPF/ES subscore, and bloating subscore where: Nausea/vomiting subscore = mean of the scores for nausea, retching, and vomiting; PPF/ES sub-score $=$ mean of the scores for stomach fullness, inability to finish meal, excessive fullness, and loss of appetite; and Bloating subscore = mean of the scores for bloating and large stomach.

Disease-specific quality of life was assessed by the Patient Assessment of Upper Gastrointestinal Disorders Quality of Life (PAGI$\mathrm{QOL}$ ) survey, which scores 30 factors from 0 (none of the time) to 5 (all of the time). ${ }^{20}$ Patients were asked how often gastrointestinal problems they may be experiencing have affected different aspects of their quality of life and well-being in the past 2 weeks. Overall PAGI-QOL scores were calculated by taking means of all subscores after reversing item scores; thus a mean PAGI-QOL score of 0 represents poor quality of life while 5 reflects the best life quality.

The Medical Outcomes Study 36-Item Short-Form Health Survey version 2 (SF-36v2) was additionally used to assess the patients' views of overall physical and mental health in the past 4 weeks. ${ }^{21}$ The eight subscales were standardized to the 1998 U.S. general population with a mean $( \pm S D)$ of $50 \pm 10$. Physical and mental health summary measures were computed. A higher score reflects higher quality of life.

\section{4 | Gastric emptying scintigraphy}

Gastric emptying scintigraphy was performed using a low-fat, egg white meal with imaging at 0, 1, 2, 4 hours after meal ingestion, as described by a published multicenter protocol ${ }^{22}$ and endorsed by the Society of Nuclear Medicine and American Neurogastroenterology and Motility Society. ${ }^{23}$ This protocol ensures standardized information about gastric emptying across sites. In addition, liquid gastric emptying in the presence of solids was assessed using Indium-111. ${ }^{24}$

Patients were instructed to stop medications that could affect gastrointestinal motility for 48 hours prior to the study and to come to the Nuclear Medicine Section in the morning after fasting overnight, that is, an 8 hour fast. Gastric emptying scintigraphy was performed using a standard low-fat, Eggbeaters ${ }^{\circledR}$ meal to measure solid emptying. ${ }^{22,23}$ The meal consisted of the equivalent of two large eggs radiolabeled with Tc-99m sulfur colloid served with two pieces of white bread and jelly. In addition, patients were given $120 \mathrm{~mL}$ water radiolabeled with In-111 diethylene triamine pentacetic acid for the measurement of liquid gastric emptying. Following ingestion of the meal, imaging was performed at 0, 1, 2, and 4 hours with the patient upright for measuring gastric emptying of Tc-labeled solids and 111-In-labeled liquids. In between imaging, patients generally sat in the nuclear medicine waiting area.

Gastric emptying was analyzed as percent of radioactivity retained in the stomach over time using the geometric center of the decaycorrected anterior and posterior counts for each time point. Gastric retention of Tc- $99 \mathrm{~m}>60 \%$ at 2 hours and/or $>10 \%$ at 4 hours was considered delayed gastric emptying of solids. Delayed gastric emptying was graded according to the gastric retention at 4 hours: mild ( $\leq 20 \%$ gastric retention at 4 hours), moderate (>20-35\%), and severe (>35\%). ${ }^{23}$ Delayed gastric emptying of liquids in the presence of solids is greater than $50 \%$ retention of $\ln -111$ at 1 hour emptying. ${ }^{24}$

\section{5 | Water load satiety testing}

A satiety test of non-caloric liquid water was performed at enrollment. The water load test is a standardized test to induce gastric distension and to evoke gastric motility responses without the complex hormonal response of a caloric test meal. On the day of testing, patients reported after fasting overnight and were instructed to drink maximal volumes of water using an opaque $150 \mathrm{~mL}$ cup over 5 minutes until they felt completely full. ${ }^{25}$ The volume of water consumed was recorded.

\subsection{Statistical methods}

Descriptive statistics (means, standard deviations, frequencies, and percentages) were used to compare subgroups of gastroparesis patients. Enrollment characteristics such as demographics, medical history, gastroparesis history, symptom severity, and quality of life were compared by etiology (idiopathic compared to diabetic). P-values were determined from Fisher's exact tests for categorical variables and $t$ tests for continuous variables. Enrollment characteristics were 
TABLE 1 Characteristics of gastroparesis patients by early satiety severity

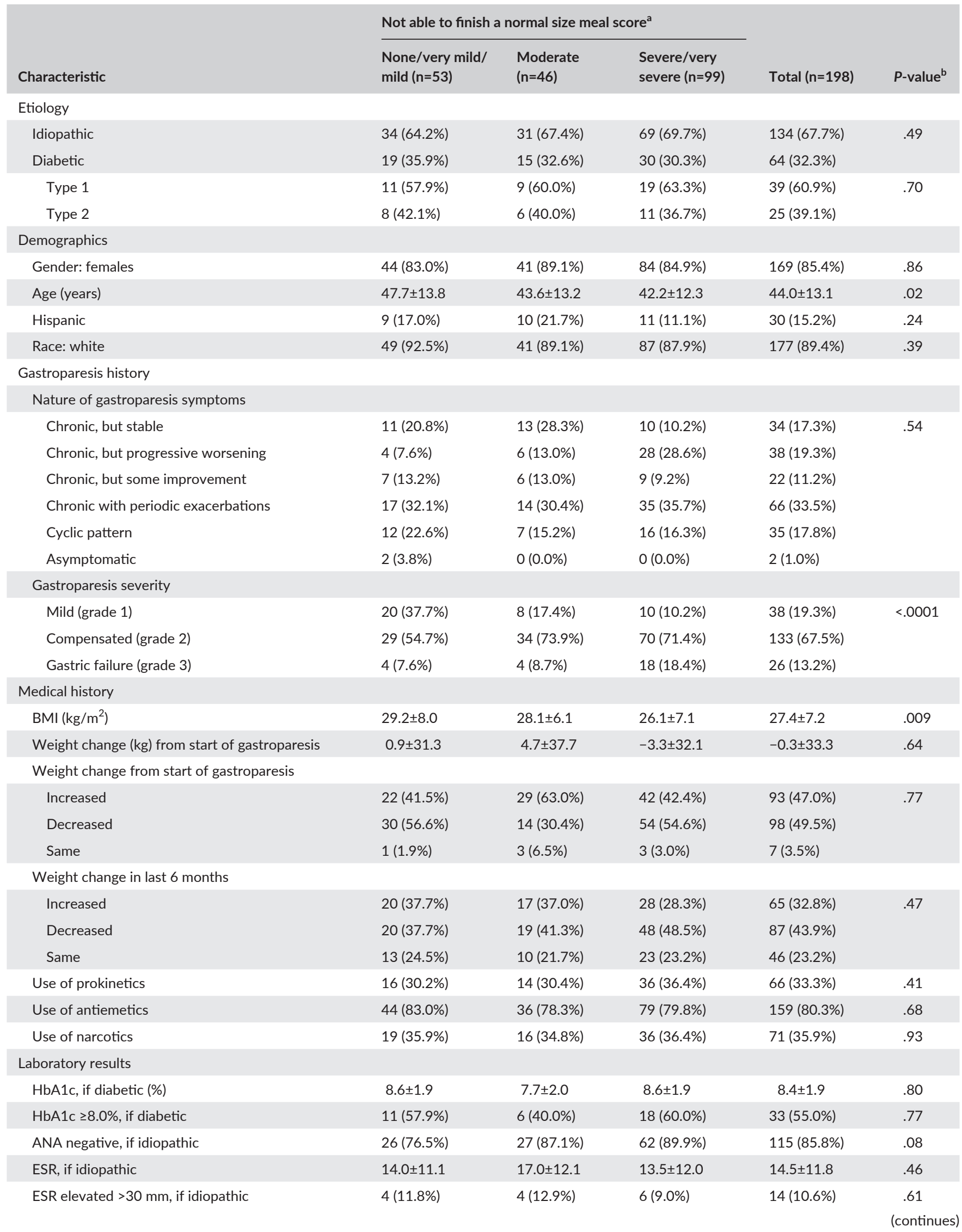


TABLE 1 (continued)

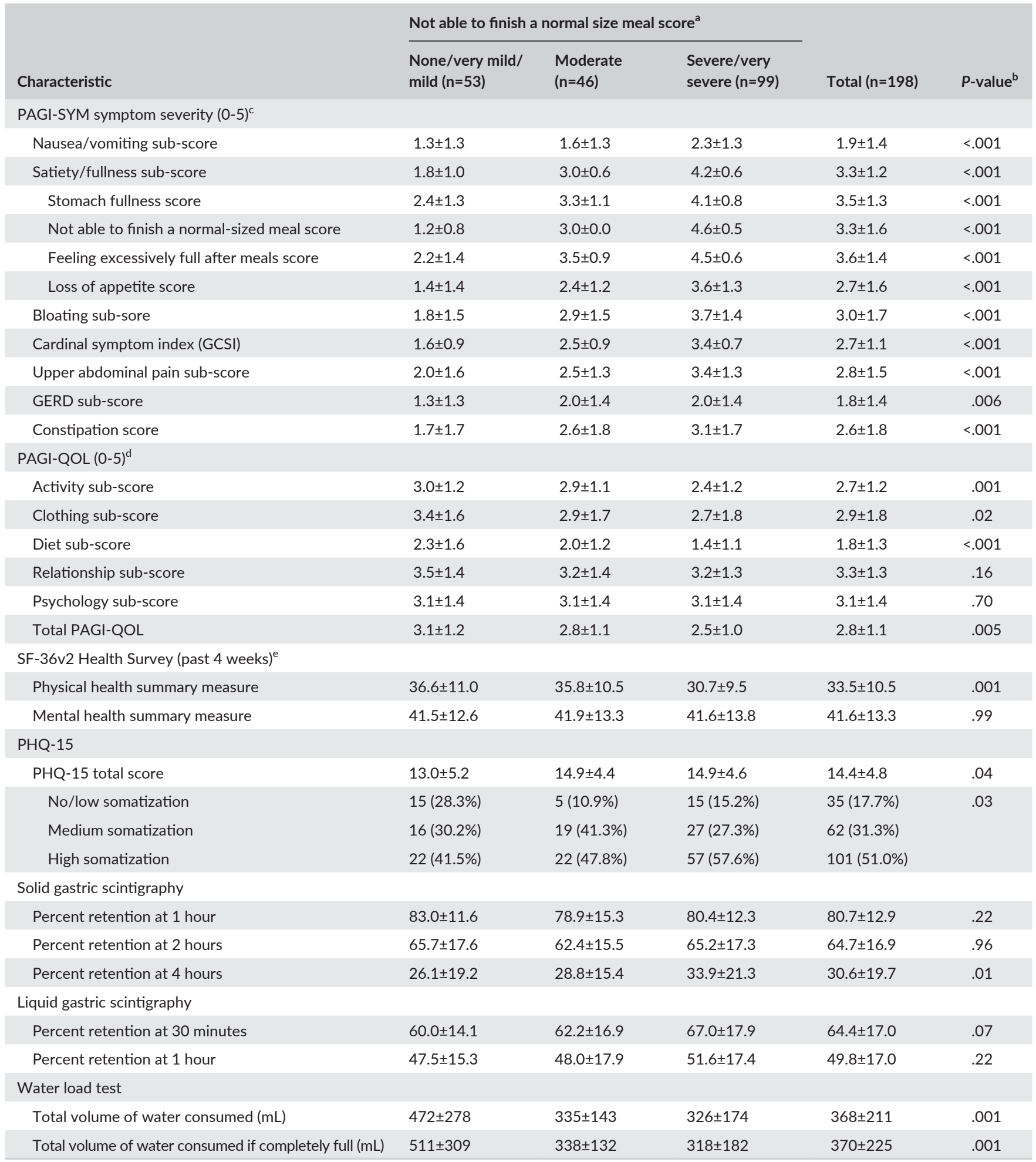

Data are means \pm standard deviations or number (percents).

${ }^{a}$ Nausea severity is a score from the Patient Assessment of Upper Gastrointestinal Disorders Symptom Severity Index (PAGI-SYM).

${ }^{\mathrm{b}}$ The significance of difference in binary variables between groups was tested with a Cochran-Armitage trend test, the significance of difference in categorical variables between groups was tested with a Mantel-Haenszel chi-squared test, and the significance of difference in continuous variables between groups was tested with a non-parametric Cuzick test for trend. All P-values are two-sided.

'Subscales derived from the Patient Assessment of Upper Gastrointestinal Disorders Symptom Severity Index (PAGI-SYM). A higher score reflects a greater severity.

${ }^{\mathrm{d}}$ Subscales derived from the Patient Assessment of Upper Gastrointestinal Disorders-Quality of Life (PAGI-QOL). Scales have been recoded so that a higher score reflects a higher QOL.

${ }^{\text {eS}}$ Scores on the Medical Outcomes Study 36-Item Short-Form Health Survey V2 (SF-36v2) standard recall were normalized to the 1998 U.S. general population with a mean $( \pm \mathrm{SD})$ of $50 \pm 10$. A higher score reflects higher QOL or better health outcome. 
also compared by the subgroups of ES severity score and PPF on the PAGI-SYM instrument (none/very mild/mild, moderate, and severe/ very severe). $P$-values were determined from a Cochran-Armitage test for trend in ES or PPF subgroups for binary variables, a MantelHaenszel chi-squared test for trend in ES or PPF subgroups for categorical variables, and a non-parametric Cuzick test for trend in ES or PPF subgroups for continuous variables. ${ }^{26}$ Multiple logistic models were selected based on Akaike Information criteria using forward selection of all possible models derived from a candidate set of 16 enrollment variables. ${ }^{27,} 28$ The resulting model for severe ES included etiology, age, solid gastric emptying percent at 4 hours, PAGI-SYM satiety/fullness sub-score, SF-36 mental score, SF-36 physical score, and PAGI-QOL score. The resulting model for severe PPF included etiology, age, race (white vs non-white), PAGI-QOL score, HbA1c\%, and the following PAGI-SYM measures: satiety/fullness sub-score, bloating sub-score, and GERD sub-score. All P-values are two-sided; values $<.05$ were considered statistically significant. Analyses were performed using methods described in SAS version 9.3 (SAS Institute) or Stata version 13.1 (StataCorp). ${ }^{29}$

\section{3 | RESULTS}

\section{1 | Patient characteristics}

198 patients with gastroparesis were evaluated: 134 patients with idiopathic gastroparesis and 64 patients with diabetic gastroparesis

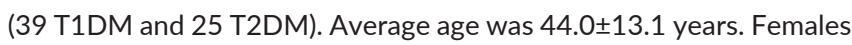
comprised the majority of patients (85.4\%). Table 1 contains other

\begin{tabular}{|c|c|c|c|c|}
\hline Characteristic & $\begin{array}{l}\text { Idiopathic } \\
(n=134)\end{array}$ & Diabetic $(n=64)$ & $\begin{array}{l}\text { Total } \\
(n=198)\end{array}$ & $P$-value \\
\hline Satiety/fullness sub-score & $3.2 \pm 1.2$ & $3.4 \pm 1.2$ & $3.3 \pm 1.2$ & .44 \\
\hline$<3$ & $46(34.3 \%)$ & $20(31.3 \%)$ & $66(33.3 \%)$ & \multirow[t]{3}{*}{.75} \\
\hline $3-3.75$ & 35 (26.1\%) & $20(31.3 \%)$ & $55(27.8 \%)$ & \\
\hline$\geq 4$ & $53(39.6 \%)$ & $24(37.5 \%)$ & 77 (38.9\%) & \\
\hline Stomach fullness & $3.4 \pm 1.3$ & $3.6 \pm 1.2$ & $3.5 \pm 1.3$ & .21 \\
\hline None-mild & $29(21.6 \%)$ & 9 (14.1\%) & $38(19.2 \%)$ & \multirow[t]{3}{*}{.42} \\
\hline Moderate & 38 (28.4\%) & $18(28.1 \%)$ & $56(28.3 \%)$ & \\
\hline Severe-very severe & 67 (50.0\%) & $37(57.8 \%)$ & $104(52.5 \%)$ & \\
\hline $\begin{array}{l}\text { Not able to finish a normal-sized } \\
\text { meal }\end{array}$ & $3.3 \pm 1.5$ & $3.2 \pm 1.6$ & $3.3 \pm 1.6$ & .55 \\
\hline None-mild & $34(25.4 \%)$ & 19 (29.7\%) & $53(26.8 \%)$ & \multirow[t]{3}{*}{.78} \\
\hline Moderate & 31 (23.1\%) & $15(23.4 \%)$ & $46(23.2 \%)$ & \\
\hline Severe-very severe & 69 (51.5\%) & $30(46.9 \%)$ & 99 (50.0\%) & \\
\hline Feeling excessively full after meals & $3.5 \pm 1.3$ & $3.8 \pm 1.4$ & $3.6 \pm 1.4$ & .21 \\
\hline None-mild & 25 (18.7\%) & $8(12.5 \%)$ & $33(16.7 \%)$ & \multirow[t]{3}{*}{.25} \\
\hline Moderate & $34(25.4 \%)$ & $12(18.8 \%)$ & $46(23.2 \%)$ & \\
\hline Severe-very severe & 75 (56.0\%) & $44(68.8 \%)$ & $119(60.1 \%)$ & \\
\hline Loss of appetite & $2.6 \pm 1.6$ & $2.9 \pm 1.6$ & $2.7 \pm 1.6$ & .39 \\
\hline None-mild & $57(42.5 \%)$ & $23(35.9 \%)$ & $80(40.4 \%)$ & \multirow[t]{3}{*}{.58} \\
\hline Moderate & 32 (23.9\%) & $15(23.4 \%)$ & $47(23.7 \%)$ & \\
\hline Severe-very severe & 45 (33.6\%) & $26(40.6 \%)$ & 71 (35.9\%) & \\
\hline \multicolumn{5}{|l|}{ Water load test } \\
\hline $\begin{array}{l}\text { Total volume of water consumed } \\
(\mathrm{mL})\end{array}$ & $354 \pm 210$ & $397 \pm 213$ & $368 \pm 211$ & .18 \\
\hline $\begin{array}{l}\text { Total volume of water consumed } \\
\text { if completely full (mL) }\end{array}$ & $362 \pm 227$ & $388 \pm 221$ & $370 \pm 225$ & .51 \\
\hline \multicolumn{5}{|l|}{ Solid gastric scintigraphy } \\
\hline Percent retention at 1 hour & $80.4 \pm 13.3$ & $81.5 \pm 12.1$ & $80.7 \pm 12.9$ & .56 \\
\hline Percent retention at 2 hours & $64.1 \pm 16.0$ & $65.8 \pm 18.9$ & $64.7 \pm 16.9$ & .51 \\
\hline Percent retention at 4 hours & $26.9 \pm 16.8$ & $38.4 \pm 22.9$ & $30.6 \pm 19.7$ & .0001 \\
\hline \multicolumn{5}{|l|}{ Liquid gastric scintigraphy } \\
\hline Percent retention at 30 minutes & $62.6 \pm 17.0$ & $67.9 \pm 16.7$ & $64.4 \pm 17.0$ & .17 \\
\hline Percent retention at 1 hour & $49.6 \pm 16.4$ & $50.2 \pm 18.3$ & $49.8 \pm 16.9$ & .88 \\
\hline
\end{tabular}

TABLE 2 Satiety/fullness sub-score and components distributions by idiopathic and diabetic gastroparesis 
demographic information. The average GCSI score was of moderate severity $(2.7 \pm 1.1)$. The majority of patients had compensated (grade 2) gastroparesis $(67.5 \%)$ with $13.2 \%$ of patients were graded as having gastric failure. At the time of enrollment in the registry, the predominant symptoms were nausea in $27.3 \%$ of patients, upper abdominal pain in $12.7 \%$ and vomiting in $5.5 \%$ of patients. Overall, the gastric emptying was moderately delayed with $30.6 \%$ retention at 4 hours, being more delayed in diabetic gastroparesis (38.4\% retention) than idiopathic gastroparesis ( $26.8 \%$ retention; $P=.0001$ ). For the diabetic patients, the average HgbA1c was $8.4 \pm 1.9 \%$ with $55.0 \%$ of the diabetic patients having $\mathrm{HgbA} 1 \mathrm{c} \geq 8.0 \%$. There was a decreased quality of life in the patients with gastroparesis most prominently with the SF-36 physical score being 33.5 compared to normal of 50

\section{2 | Early satiety}

Early satiety was severe or very severe in 99 of 198 (50\%) patients (Table 1). Increasing severity of ES was associated with increasing gastroparesis severity $(P<.0001)$. Increasing severity of ES was associated with other gastroparesis symptoms including nausea/vomiting subscore, satiety/early fullness subscore, bloating subscore, and individual symptom severity scores for PPF, loss of appetite and upper abdominal pain, and GERD subscore. Increasing severity of ES was associated with decreased BMI $(P=.009)$, decreased quality of life from PAGI-QOL $(P=.005)$ and SF-36 physical health $(P=.001)$. Increasing severity of ES was associated with increasing gastric retention of a solid meal at 4 hours $(P=.01)$ and decrease in volume consumed during the water load test $(P=.001)$.

Severity scores for ES were similar between idiopathic and diabetic gastroparesis (3.3 vs 3.2) (Table 2). Other symptoms such as loss of appetite and stomach fullness and the GCSI subscore for satiety/fullness were similar between idiopathic and diabetic gastroparesis.
Logistic regression analysis showed that severe/very severe ES was associated with increasing bloating subscore $(P<.001)$, increasing upper abdominal pain subscore $(P=.002)$, and SF-36 mental component $(P=.02)$, and increased GE retention at 4 hours $(P=.007)$, and decreased BMI $(P=.001)$ (Table 3).

\section{3 | Postprandial fullness}

Postprandial fullness was severe or very severe in 119 of 198 (60\%) patients (Table 4). Increasing severity of PPF was associated with other gastroparesis symptoms including nausea/vomiting subscore, satiety/early fullness subscore, and bloating subscore and individual symptoms of ES, loss of appetite, upper abdominal pain, and GERD subscore. Increasing severity of PPF was associated with gastroparesis severity $(P=.003)$, decreased quality of life from PAGI-QOL $(P=.006)$ and SF-36 physical health $(P=.006)$, but not body weight $(P=.30)$. Increasing severity of PPF was associated with increasing gastric retention of a solid meal at 4 hours $(P=.01)$ and a decrease in the volume consumed during the water load test $(P=.01)$.

Severity scores for PPF (3.5 vs 3.8) were similar between idiopathic and diabetic gastroparesis (Table 2).

Logistic regression analysis showed that severe/very severe PPF was associated with retching severity $(P=.01)$, bloating subscore $(P<.001)$, upper abdominal pain subscore $(P=.001)$, and decreased BMI (Table 5).

\section{4 | DISCUSSION}

This study shows that ES and PPF are common symptoms in gastroparesis, both in diabetic and idiopathic gastroparesis. Many patents have severe or very severe ES (50\% of patients) and PPF (60\% of patients).

TAB LE 3 Logistic regression analyses of baseline predictors on severe early satiety ${ }^{\mathrm{a}}$ in idiopathic and diabetic gastroparetics ( $\mathrm{n}=196$ )

\begin{tabular}{|c|c|c|c|c|}
\hline Baseline characteristic & \multicolumn{2}{|c|}{ Unadjusted analyses } & \multicolumn{2}{|l|}{ Adjusted analysis } \\
\hline Etiology (diabetic vs idiopathic) & $0.83(0.46,1.51)$ & .54 & $0.47(0.19,1.18)$ & .11 \\
\hline Gender (female vs male) & $0.92(0.42,2.03)$ & .84 & $0.29(0.10,0.88)$ & .03 \\
\hline $\mathrm{BMI}\left(\mathrm{kg} / \mathrm{m}^{2}\right)$ & $0.95(0.91,0.99)$ & .01 & $0.91(0.86,0.96)$ & .001 \\
\hline PAGI-SYM, bloating score & $1.75(1.42,2.16)$ & $<.001$ & $1.98(1.48,2.64)$ & $<.001$ \\
\hline PAGI-SYM, upper abdominal pain sub-score & $1.83(1.46,2.29)$ & $<.001$ & $1.55(1.17,2.07)$ & .002 \\
\hline PAGI-SYM, GERD sub-score & $1.19(0.97,1.46)$ & .10 & $0.77(0.57,1.03)$ & .08 \\
\hline SF-36, mental score & $1.00(0.98,1.02)$ & .96 & $1.03(1.01,1.05)$ & .02 \\
\hline
\end{tabular}

aSevere early satiety defined as "severe" or "very severe" inability to finish a normal-sized meal score on the PAGI-SYM instrument.

bUnadjusted odds ratios, $95 \%$ confidence limits, $P$-values determined from logistic regression models of severe early satiety on each predictor.

${ }^{c}$ Adjusted odds ratios, $95 \%$ confidence limits, $P$-values were determined from a multiple logistic regression analyses of severe early satiety using all baseline predictors indicated. This model was determined from Akaike Information criteria (AIC) best subsets variable selection using a candidate set of baseline variables: gender, age at enrollment, etiology, race, BMI, SF-36 physical score, SF-36 mental score, PAGI-QOL total score, solid GES 2 hour retention percent, solid GES 4 hour retention percent, and the following PAGI-SYM items: nausea score, vomiting score, retching score, bloating score, stomach visibly larger score, upper abdominal pain sub-score, and GERD sub-score. 
TAB LE 4 Characteristics of gastroparesis patients by postprandial fullness severity

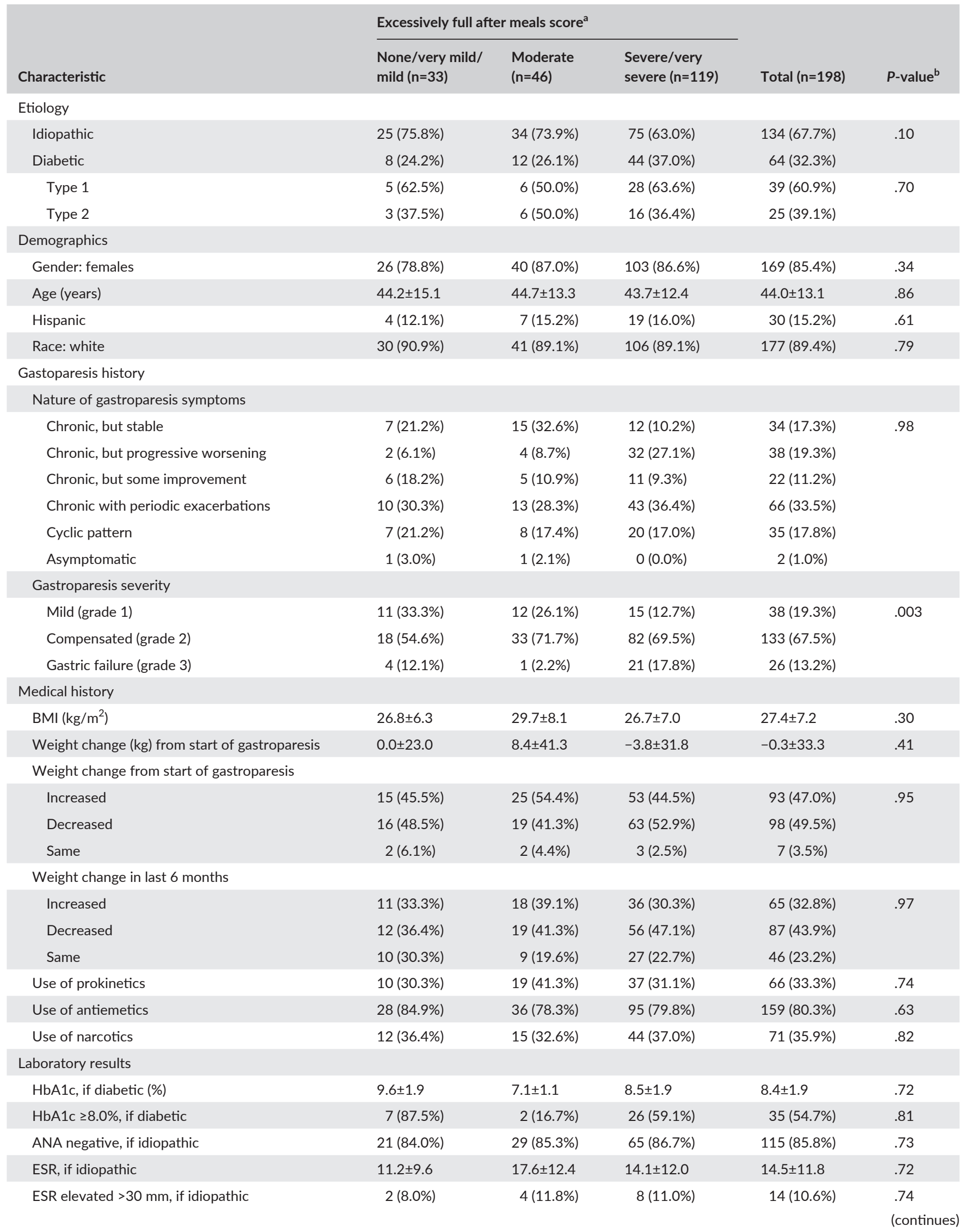


TABLE 4 (continued)

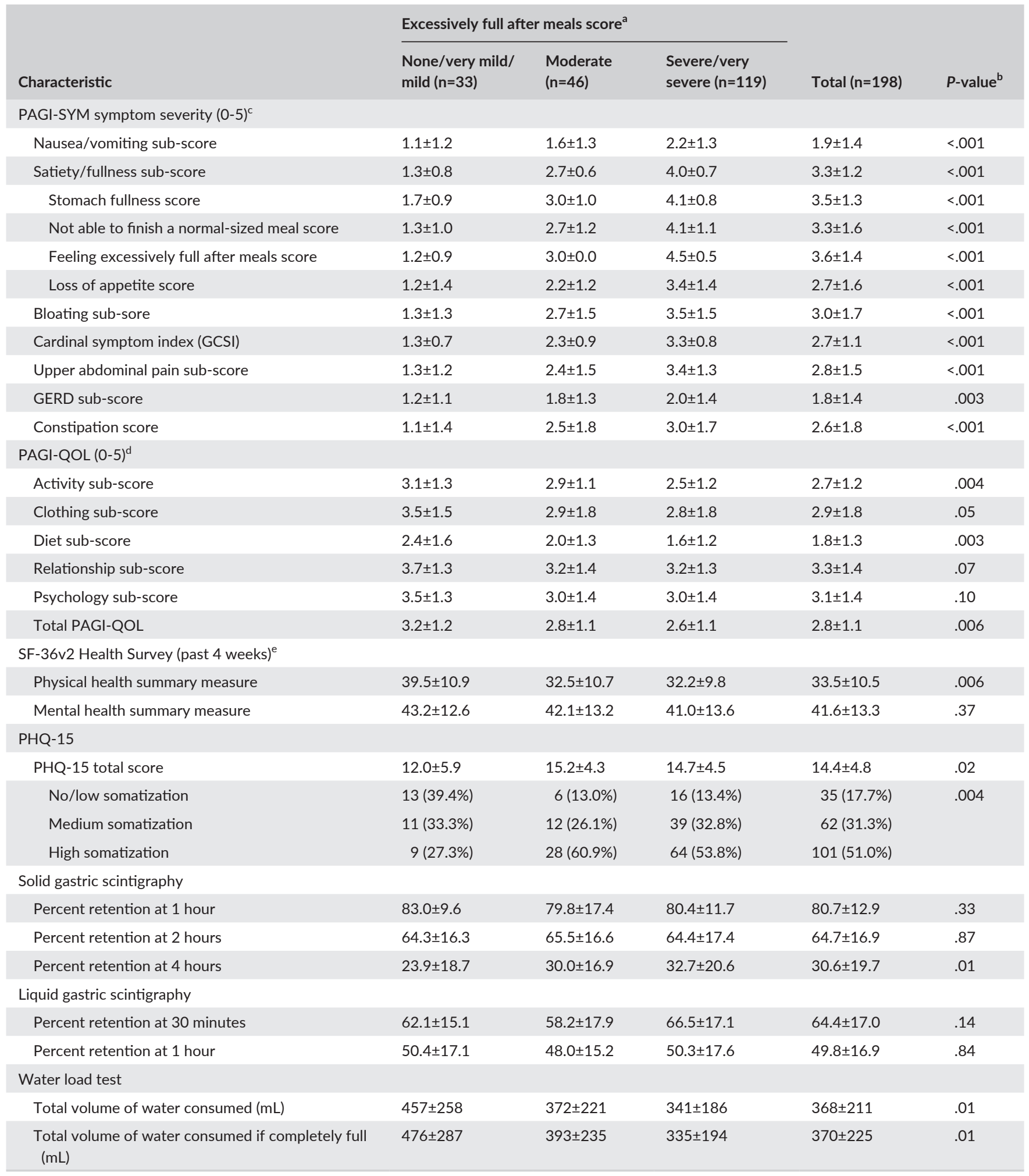

Data are means \pm standard deviations or number (percents).

${ }^{a}$ Nausea severity is a score from the Patient Assessment of Upper Gastrointestinal Disorders Symptom Severity Index (PAGI-SYM).

${ }^{\mathrm{b}}$ The significance of difference in categorical variables between groups was tested with Fisher's exact test or chi-squared test. Continuous variables were analyzed with ANOVA. All P-values are two-sided.

'Subscales derived from the Patient Assessment of Upper Gastrointestinal Disorders Symptom Severity Index (PAGI-SYM). A higher score reflects a greater severity.

${ }^{\mathrm{d}}$ Subscales derived from the Patient Assessment of Upper Gastrointestinal Disorders-Quality of Life (PAGI-QOL). Scales have been recoded so that a higher score reflects a higher QOL.

eScores on the Medical Outcomes Study 36-Item Short-Form Health Survey V2 (SF-36v2) standard recall were normalized to the 1998 U.S. general population with a mean $( \pm \mathrm{SD})$ of $50 \pm 10$. A higher score reflects higher $\mathrm{QOL}$ or better health outcome. 


\begin{tabular}{|c|c|c|c|c|}
\hline \multirow[b]{2}{*}{ Baseline characteristic } & \multicolumn{2}{|c|}{ Unadjusted analyses } & \multicolumn{2}{|l|}{ Adjusted analyses } \\
\hline & OR (CI) & $P^{\mathrm{b}}$ & OR (Cl) & $P^{c}$ \\
\hline Etiology (diabetic vs idiopathic) & $1.73(0.92,3.25)$ & .09 & $2.13(0.92,4.91)$ & .08 \\
\hline $\mathrm{BMI}\left(\mathrm{kg} / \mathrm{m}^{2}\right)$ & $0.97(0.93,1.00)$ & .08 & $0.94(0.89,0.99)$ & .01 \\
\hline PAGI-SYM, nausea score & $1.54(1.27,1.88)$ & $<.001$ & $2.15(0.92,5.06)$ & .08 \\
\hline PAGI-SYM, vomiting score & $1.39(1.14,1.68)$ & .001 & $1.09(0.69,1.73)$ & .72 \\
\hline PAGI-SYM, retching score & $1.28(1.06,1.55)$ & .01 & $0.52(0.31,0.87)$ & .01 \\
\hline $\begin{array}{l}\text { PAGI-SYM, stomach visibly } \\
\text { larger score }\end{array}$ & $2.03(1.60,2.57)$ & $<.001$ & $1.62(1.28,2.04)$ & $<.001$ \\
\hline $\begin{array}{l}\text { PAGI-SYM, upper abdominal } \\
\text { pain sub-score }\end{array}$ & $1.68(1.40,2.02)$ & $<.001$ & $1.68(1.27,2.21)$ & $<.001$ \\
\hline
\end{tabular}

TABLE 5 Logistic regression analyses of baseline predictors on severe postprandial fullness ${ }^{\mathrm{a}}$ in idiopathic and diabetic gastroparetics $(n=198)$

aSevere postprandial fullness defined as "severe" or "very severe" excessively full after meals score on the PAGI-SYM instrument.

bUnadjusted odds ratios, $95 \%$ confidence limits, $\mathrm{P}$ values determined from logistic regression models of severe fullness on each predictor.

${ }^{\mathrm{c}}$ Adjusted odds ratios, $95 \%$ confidence limits, $P$ values were determined from a multiple logistic regression analyses of severe fullness using all baseline predictors indicated. This model was determined from Akaike Information criteria (AIC) best subsets variable selection using a candidate set of baseline variables: gender, age at enrollment, etiology, race, BMI, SF-36 physical score, SF-36 mental score, PAGI-QOL total score, solid GES 2 hour retention percent, solid GES 4 hour retention percent, and the following PAGI-SYM items: nausea score, vomiting score, retching score, bloating score, stomach visibly larger score, upper abdominal pain sub-score, and GERD sub-score.

Early satiety and PPF severity are associated with other measures of gastroparesis severity including overall gastroparesis severity, body weight, quality of life, gastric emptying, water load testing. These characteristics of ES and PPF to gastroparesis severity, quality of life, and physiologic abnormalities make ES and PPF important symptoms characterizing gastroparesis.

Gastric accommodation as assessed by barostat or SPECT scanning, have been correlated with the symptom of ES. ${ }^{11,30}$ The water load test was used in this multicenter study as a satiety test of a non-caloric water liquid meal. ${ }^{25}$ This standardized test induces gastric distension and evokes gastric motility responses without the complex hormonal response of a caloric test meal. Prior studies have shown that patients with functional dyspepsia ingested smaller volumes of water than normal controls ( $358 \mathrm{~mL}$ vs $557 \mathrm{~mL}) .^{25}$ Our patients with gastroparesis ingested on average low volumes of water $(368 \mathrm{~mL})$. Our study shows that increasing symptoms of ES and PPF are objectively associated with decreased volume consumed during the water load test.

The symptoms of ES and PPF are both associated with delayed gastric emptying. Increasing severity of ES and PPF are associated with increased gastric retention at 4 hours. In multivariate analysis, ES but not PPF was associated with impaired gastric emptying. Other studies have been able to relate nausea, vomiting, and PPF to delayed gastric emptying. ${ }^{12}$

Functional dyspepsia is diagnosed by having symptoms of PPF, early satiation, epigastric pain/burning. Symptoms of gastroparesis overlap with those of functional dyspepsia. This is not surprising since one (gastroparesis) involves gastric symptoms with objective delay in gastric emptying, whereas the other (functional dyspepsia) is a symptom only based disorder. Our gastroparesis consortium has shown that nearly $85 \%$ of gastroparesis patients meet the definition of functional dyspepsia, primarily postprandial distress syndrome
(PDS).$^{15}$ Early satiety and PPF are also prevalent in patients with the PDS form of functional dyspepsia, since in PDS, meal ingestion triggers symptoms. ${ }^{31}$

Early satiety and PPF symptoms appear to capture different pathophysiologic aspects of gastroparesis: ES - impaired proximal gastric (fundic) function and PPF - impaired distal gastric (antral) function. This study shows that the characteristics of ES overlap with PPF. The presence of ES vs PPF does not differentiate between other symptoms in gastroparesis. Early satiety and PPF severity are significantly associated with other gastroparesis symptoms including nausea/vomiting subscore and bloating subscore and measures of gastroparesis severity, and quality of life, gastric emptying, water load testing. On multivariable analysis, significant relationships were found between ES with bloating and upper abdominal pain and between PPF with retching, stomach distension, and upper abdominal pain. Pathophysiologically, both ES and PPF are related to delayed gastric emptying and impairments of the water load test. Although on multivariate analysis, ES but not PPF was associated with impaired gastric emptying. It is perhaps not surprising that the severity of ES is associated with PPF: with ES, the patients becomes full early with eating, this fullness may persist after eating leading to PPF. These two symptoms of ES and PPF may not be reliably distinguished by patients. In a recent study that looked at quality of life in gastroparesis patients, ${ }^{32}$ four of the five symptoms suggested by the FDA guidance document of gastroparesis ${ }^{5}$ were significantly related to impaired QOL, namely, nausea, vomiting, ES, and upper abdominal pain. Interestingly, PPF was not an independent predictor of QOL physical health. This suggests that, of the two symptoms, ES might be the preferable symptom to ask about.

In summary, the results of this study bring to forefront the notion that ES and PPF are common symptoms in gastroparesis that often judged by the patients to be severe. Early satiety and PPF severity are 
significantly associated with other measures of gastroparesis severity, and quality of life, gastric emptying, body weight, and water load testing. These relationships of ES and PPF to gastroparesis severity, quality of life, and gastric emptying make these important symptoms characterizing gastroparesis and important symptoms to follow in treating patients with gastroparesis.

\section{ACKNOWLEDGMENTS}

The Gastroparesis Clinical Research Consortium (GpCRC) is supported by the National Institute of Diabetes and Digestive and Kidney Diseases (NIDDK) (grants U01DK073975, U01DK073983, U01DK073985, U01DK074007, U01DK073974, U01DK074008).

\section{CONFLICTS OF INTEREST}

No conflicts of interest exist.

\section{AUTHOR CONTRIBUTION}

HPP study conceptualization, patient recruitment, data interpretation, writing manuscript; EH statistical analysis, data interpretation, writing manuscript; WLH, KLK, LN, WJS, TLA, PJP, study conceptualization, patient recruitment, revising manuscript; GF and FH study conceptualization, revising manuscript; RWM, IS, and JC patient recruitment, revising manuscript; LM study conceptualization, study implementation; JT study conceptualization, statistical analysis, data interpretation, revising manuscript.

\section{REFERENCES}

1. Parkman HP, Hasler WL, Fisher RS. American Gastroenterological Association technical review on the diagnosis and treatment of gastroparesis. Gastroenterology. 2004;127:1592-1622.

2. Soykan I, Sivri B, Sarosiek I, et al. Demography, clinical characteristics, psychological and abuse profiles, treatment, and long-term follow-up of patients with gastroparesis. Dig Dis Sci. 1998;43:2398-2404.

3. Nguyen LA, Snape WJ Jr. Clinical presentation and pathophysiology of gastroparesis. Gastroenterol Clin North Am. 2015;44:21-30.

4. Revicki DA, Camilleri M, Kuo B, Szarka LA, McCormack J, Parkman HP. Evaluating symptom outcomes in gastroparesis clinical trials: validity and responsiveness of the Gastroparesis Cardinal Symptom Index-Daily Diary (GCSI-DD). Neurogastroenterol Motil. 2012;24: 456-463.

5. Hui R. Gastroparesis: Clinical Evaluation of Drugs for Treatment: Guidance for Industry. Food and Drug Administration Center for Drug Evaluation and Research (CDER); 2015. Available at: http://www.fda. gov/downloads/drugs/guidancecomplianceregulatoryinformation/ guidances/ucm455645.pdf. Accessed October 6, 2016.

6. Kelly KA. Gastric emptying of liquids and solids: roles of proximal and distal stomach. Am J Physiol. 1980;239:G71-G76.

7. Collins PJ, Horowitz M, Chatterton BE. Proximal, distal and total stomach emptying of a digestible solid meal in normal subjects. Br J Radiol. 1988;61:12-18.

8. Azpiroz F. Control of gastric emptying by gastric tone. Dig Dis Sci. 1994;39:18S-19S.

9. Troncon LE, Bennett RJ, Ahluwalia NK, Thompson DG. Abnormal intragastric distribution of food during gastric emptying in functional dyspepsia patients. Gut. 1994;35:327-332.
10. Tack J, Piessevaux H, Coulie B, et al. Role of impaired gastric accommodation to a meal in functional dyspepsia. Gastroenterology. 1998;115:1346-1352.

11. Karamanolis G, Caenepeel P, Arts J, Tack J. Determinants of symptom pattern in idiopathic severely delayed gastric emptying: gastric emptying rate or proximal stomach dysfunction? Gut. 2007;56:29-36.

12. Stanghellini V, Tack J. Gastroparesis: separate entity or just a part of dyspepsia? Gut. 2014;63:1972-1978.

13. Stanghellini $\mathrm{V}$, Tosetti $\mathrm{C}$, Paternico A, et al. Risk indicators of delayed gastric emptying of solids in patients with functional dyspepsia. Gastroenterology. 1996;110:1036-1042.

14. Sarnelli G, Caenepeel P, Geypens B, Janssens J, Tack J. Symptoms associated with impaired gastric emptying of solids and liquids in functional dyspepsia. Am J Gastroenterol. 2003;98:783-788.

15. Parkman HP, Yates $K$, Hasler WL, et al. Clinical features of idiopathic gastroparesis vary with sex, body mass, symptom onset, delay in gastric emptying, and gastroparesis severity. Gastroenterology. 2011;140:101-115.

16. Hasler WL, Parkman HP, Wilson LA, et al. Psychological dysfunction is associated with symptom severity but not disease etiology or degree of gastric retention in patients with gastroparesis. Am J Gastroenterol. 2010;105:2357-2367.

17. Abell TL, Bernstein VK, Cutts T, et al. Treatment of gastroparesis: a multidisciplinary clinical review. Neurogastroenterol Motil. 2006;18:263-283.

18. Rentz AM, Kahrilas P, Stanghellini V, et al. Development and psychometric evaluation of the patient assessment of upper gastrointestinal symptom severity index (PAGI-SYM) in patients with upper gastrointestinal disorders. Qual Life Res. 2004;13:1737-1749.

19. Revicki DA, Rentz AM, Dubois D, et al. Development and validation of a patient-assessed gastroparesis symptom severity measure: the Gastroparesis Cardinal Symptom Index. Aliment Pharmacol Ther. 2003;18:141-150.

20. De la Loge C, Trudeau E, Marquis P, et al. Cross-cultural development and validation of a patient self-administered questionnaire to assess quality of life in upper gastrointestinal disorders: the PAGI-QOL. Qual Life Res. 2004;13:1751-1762.

21. Ware JE, Kosinski M, Dewey JE. How to Score Version 2 of the SF-36® Health Survey. Lincoln, RI: QualityMetric Incorporated; 2000.

22. Tougas G, Eaker EY, Abell TL, et al. Assessment of gastric emptying using a low fat meal: establishment of international control values. Am J Gastroenterol. 2000;95:1456-1462.

23. Abell TL, Camilleri M, Donohoe K, etal. Consensus recommendations for gastric emptying scintigraphy. Am J Gastroenterol. 2008;103:753-763.

24. Sachdeva P, Malhotra N, Pathikonda M, et al. Gastric emptying of solids and liquids for evaluation for gastroparesis. Dig Dis Sci. 2011;56:1138-1146.

25. Koch KL, Hong SP, Xu L. Reproducibility of gastric myoelectrical activity and the water load test in patients with dysmotility-like dyspepsia symptoms and in control subjects. J Clin Gastroenterol. 2000;31:125-129.

26. Agresti A. Categorical Data Analysis. New York, NY: John Wiley \& Sons, Inc.; 1990.

27. Akaike H. A new look at the statistical model identification. IEEE Trans Autom Control. 1974;19:716-723.

28. Hosmer D, Lemeshow S. Applied Logistic Regression, 2nd edn. New York, NY: John Wiley \& Sons, Inc.; 2000.

29. SAS Institute, Inc. SAS Software, Version 9.3 of the SAS System for Windows. Cary, NC, 2002-2010. Stata Statistical Software: Release 12. College Station, TX: StataCorp LP; 2011.

30. Vijayvargiya P, Camilleri M, Shin A, Breen M, Burton D. Simplifying the measurement of gastric accommodation using SPECT. Neurogastroenterol Motil. 2013;25:542-546.

31. Stanghellini V, Chan FK, Hasler WL, et al. Gastroduodenal disorders. Gastroenterology. 2016;150:1380-1392.

32. Yu D, Ramsey FV, Norton W, Norton N, Gaetano T, Parkman HP. Impaired quality of life in gastroparesis: relationships to symptoms of gastroparesis. Gastroenterology 2016;150:S718. 